The Effects of First-Year Residence Hall Roommate Assignment Policy on Interactional Diversity and Perceptions of the Campus Environment

\author{
Kevin Fosnacht \\ Robert M. Gonyea \\ Polly A. Graham \\ Indiana University Bloomington
}

Sarah Hurtado

University of Denver

Corresponding author:

Kevin Fosnacht

Indiana University Center for Postsecondary Research

1900 East Tenth Street, Suite 419

Bloomington, IN 47406-7512

812-856-1961

kfosnach@indiana.edu

Authors' note: This research was funded by the ACUHO-I Research and Educational Foundation. Paper was presented at the 2018 annual meeting of the Association for the Study of Higher Education. 


\title{
The Effects of First-Year Residence Hall Roommate Assignment Policy on Interactional Diversity and Perceptions of the Campus Environment
}

\begin{abstract}
A heterogenous student body is valued in part because diverse interactions among students help create educated and competent citizens and promotes student learning and development. Campus housing is a primary area for diverse interactions as students navigate living with individuals who differ from themselves. This study investigates how the roommate assignment process influences interactional diversity and perceptions of the campus environment for first-year students living on campus, and if these relationships differ by race/ethnicity. On average, students whose roommates were assigned by the institution (as opposed to choosing their own roommates) had a trivial increase in interactional diversity, but perceived a less supportive campus environment. However, the latter relationship differed by race. In particular, Asian, Black and multiracial students who were assigned roommates by the institution perceived a less welcoming campus environment than their same-race peers who chose their roommates. This difference was not observed for White students.
\end{abstract}

Keywords: Residence life, residence halls, roommates, interactional diversity, campus environment 


\section{The Effects of First-Year Residence Hall Roommate Assignment Policy on Interactional Diversity and Perceptions of the Campus Environment}

\section{Introduction}

Diversity has become a core value within the academy, evidenced from mission statements to recruiting materials to the legal battles over affirmative action. A heterogeneous student body is valued in part because interactional diversity helps create educated and competent citizens and influences student learning (Antonio, Chang, Hakuta, Kenny, Levin, \& Milem, 2004; Chang, 1999; Gurin, Dey, Hurtado, \& Gurin, 2002; Gurin, Nagda, \& Lopez, 2004). Campus housing is a primary area where the abstract goal of diverse interactions becomes a concrete reality - often for the first time - as students navigate living with and near individuals who differ from themselves in significant ways such as by race, religion, politics, country of origin, and socio-economic status. For example, a recent study found that White students who were assigned a different-race roommate had more friends (other than the roommate) from their roommate's race than those who were assigned to room with other White students (Mark \& Harris, 2012).

While housing policies vary, many bachelor's degree-granting institutions require living on campus for the first year to provide students more convenient access to campus programming and support services designed for their success. Thus, by negotiating this new environment students' housing experiences represent a prime way to increase learning and development regarding diversity. Consequently, this study investigates whether the roommate assignment process influences students' interactional diversity and perceptions of the campus environment. 
Furthermore, we examine if the relationship between being assigned a roommate and these outcomes varies by students' racial/ethnic backgrounds.

\section{Literature Review}

Before the 1980's, roommate assignments were mostly random, based on bare minimum preferences such as whether or not a student was a smoker. In 1980, an important study by Hallisey and colleagues highlighted the importance of roommates in students' choices to move off-campus. This work prompted student development personnel to develop better strategies for roommate assignments to help students realize the benefits of living on campus. Room assignment processes attempted to minimize negative roommate interactions by pairing residents with similar preferences (Molina, et al., 2015). Hallisey and colleagues (1980) argued that roommate compatibility is a complex topic and that students were in a better position than administrators to identify a good match. Consequently, for the past four decades most students have been able to choose their roommates. For those who did not choose a roommate, many institutions have made assignments based on student preferences like sleeping schedules and tolerance for noise.

In recent years, the rise of social media and online roommate matching sites have changed how students find roommates (Conley, 2011; Li-A-Ping, n.d.; Ward, 2008; Washington Post Editors, 2010), and the proportion of incoming students choosing to live with a specific student has increased (Bauer-Wolf, 2018). Today's digital native students use the internet to meet other matriculating students from beyond their own high school or hometown. Using social media like Facebook or dedicated roommate matching sites like Roomsurf.com, prospective oncampus residents can search on their own for a good match (Conley, 2011; Li-A-Ping, n.d.; Washington Post Editors, 2010). This trend has some universities worried about the impact on 
first-year growth and development, as students often select roommates who are like themselves.

For example, Duke University recently decided to take back the decision-making power, requiring all first-year students to be assigned roommates by the university (Bauer-Wolf, 2018). As their vice president for student affairs explained, "We're being very deliberate about the kind of first-year experience that we think would best suit our students — an experience that really is about engaging with difference and opening their eyes to opportunities, and meeting entirely different people than the ones they grew up with or went to high school with. We just thought that [randomly selected roommates] would be a better approach" (Simon, 2018, para. 10).

While policies like Duke's may be well-intentioned, previous research indicates that the effects of interactional diversity vary by student characteristics (Bowman, 2010; Gurin et al., 2004). For example, the benefits of diversity courses were found to vary by wealth and race, with wealthier and White students experiencing higher gains when taking two or more diversity courses (Bowman, 2010). Such studies caution those implementing new diversity-related policies to ask questions about equity of impact.

Other research suggests that microaggressions are frequent in social spaces like residence halls, where diverse interactions may actually harm historically marginalized populations (e.g., Solórzano, Allen, \& Carroll, 2002; Solórzano, Ceja, \& Yosso, 2000; Yosso, Smith, Ceja \& Solórzano, 2009). A study conducted at selective universities identified racial microaggressions that Latina/o students faced, including interpersonal microaggressions and racial jokes, which led their participants to feel as if they did not belong at the institution (Yosso et al., 2009). What led to success for these students was not integration, but rather creating safe counterspaces that embraced their cultural norms rather than questioning them or mocking them. The rise in counterspaces highlights the need for marginalized groups to share areas distinct from the 
dominant culture. Yet, policies restricting roommate choice can prevent residence hall rooms from being truly safe spaces (Solórzano et al., 2002).

The extant literature suggests that while interactional diversity in college is associated with positive outcomes, institutional decision-makers need to assess whether different types of students benefit and to what degree. If diversity initiatives primarily benefit historically privileged groups, especially at the expense of historically marginalized groups, creative and informed action should be taken toward more socially just policy and practice.

\section{Theory}

Our study draws upon two theories for guidance. The first is social structure theory which helps us understand the effect of roommate matching on interactional diversity. The second, Museus' Culturally Engaging Campus Environments model addresses how students' perceptions of the campus environment affect sense of belonging as it relates to cultural drivers.

Social structure theory suggests that individuals are more likely to interact when three conditions are present: homophily, proximity, and transitivity (Mark \& Harris, 2012). Homophily means that people connect socially with others who are like themselves in terms of a given characteristic such as age, income, race/ethnicity, education, and so on (Blau, 1977). Proximity means that people are more likely to connect socially with others who are nearby and accessible, such as by neighborhood, school, or work location, thus making it more likely that people encounter each other (Festinger, Schachter, \& Back, 1950). Transitivity is a principle in friendship networks that means that individuals are likely to hang out with the friends of those with whom they are already friends (Holland \& Lienhardt, 1972). In other words, if person A and person $\mathrm{B}$ are friends, and persons $\mathrm{B}$ and $\mathrm{C}$ are friends, then person $\mathrm{A}$ is more likely to connect socially with person $\mathrm{C}$. 
Applying these principles to the current study, we assumed that roommates who were friends before they chose to live together - including those who met through social media applications - are likely to share one or more characteristics (homophily). In contrast, we also assumed institution-assigned roommates were more likely to be different from each other in one or more characteristics (as most modern matching practices focus on lifestyle issues like sleep habits and cleanliness, not background characteristics). Next, students who shared living quarters, of course, had the advantage of proximity and were therefore likely to engage in additional social connections outside of the room, such as going out for meals, social activities, and other campus events (proximity). Finally, roommates are also assumed to have connected socially with the friends of their roommates (transitivity). Because those friends are likely to be similar in some characteristics with the roommate (homophily), in cases where roommate A is of a different race or income status, roommate B could connect socially with others who are different. Therefore, social structure theory guides us to expect that roommates who were matched by the institution would have more frequent interactions with diversity.

Additionally, there is a need to understand the factors that influence a students' sense of belonging. There is evidence that a student's perception of the campus climate is related to the ways they experience sense of belonging (Museus, Yi, \& Saelua, 2017). For example, Hurtado and Carter (1997) found that when Latino students perceived a more hostile campus climate their sense of belonging decreased. Similarly, when Filipino American students experienced pressure to disconnect from their cultural community and assimilate to the campus culture they felt a lower sense of belonging (Museus \& Maramba, 2011). The Culturally Engaging Campus Environments (CECE) Model theorizes that when a student has access to a culturally engaging campus environment they experience greater sense of belonging and other positive outcomes 
(Museus, 2014). In the CECE Model, there are nine indicators of a culturally engaging campus environment: cultural relevance, cultural familiarity, culturally relevant knowledge, cultural community service, meaningful cross-cultural engagement, culturally validating environments, cultural responsiveness, collectivist cultural orientations, humanized educational environments, and holistic support (Museus, 2014). Although all nine indicators within the CECE model positively influence students' sense of belonging, there are some that are particularly relevant to this study. First, cultural familiarity exists when students have the opportunity to interact with others on campus (e.g., peers, faculty) who understand their cultural background. Second, meaningful cross-cultural engagement reflects the extent to which students can "engage in positive and purposeful interactions with peers from disparate cultural origins" (Museus, 2014, p. 211). An important element of this indicator is that these interactions are meaningful; simply having interactions with students who are different from themselves is not enough if these interactions results in microaggressions, exclusion, etc. Thus, applying these ideas, we would expect that roommates who were matched by the institution would be less likely to live with someone who is familiar with their own culture, but - perhaps consistent with social structure theory - would be more likely to have opportunities for meaningful cross-cultural engagement.

\section{Research Questions}

Guided by the theory above, we investigated the following research questions:

1. How does the method of roommate selection influence first-year students' interactional diversity and perceptions of the college environment?

2. Does the relationship between the method of roommate selection and these outcomes vary by race/ethnicity?

\section{Methods}




\section{Data}

We utilized data from the 2018 administration of the National Survey of Student Engagement (NSSE), a large multi-institution study that examines the engagement of bachelor's degree-seeking students in effective educational activities, perceptions of the campus environment, and other topics associated with their learning and development. Specifically, our data come from 76 institutions that participated in a special housing study investigating how student living conditions influence student outcomes. The response rate for the first-year sample from the housing study (including students who lived off campus) was $22.4 \%$. Due to our focus on roommate selection, we narrowed the sample to 14,401 first-year students who reported that they lived on campus with at least one roommate.

Seventy percent of the respondent sample was female. White students comprised $61 \%$ of respondents, African Americans were 12\%, Latinx were 6\%, Asian and Pacific Islanders were $5 \%$, multi-racial students were $9 \%$, international students made up 5\%, and less than one percent were grouped in an 'other' category. Slightly more a third of the sample was first-generation, nearly all were enrolled full time, and the average age was 18.4 years. A majority of respondents were enrolled at public institutions, with slightly less than half at doctoral universities, about a quarter at masters-granting institutions, and $13 \%$ at baccalaureate colleges. One in twenty students attended an institution rated less competitive, $62 \%$ attended institutions rated competitive, $19 \%$ were enrolled at institutions rated very competitive, and the remaining proportion attended institutions rated highly to most competitive.

Three NSSE Engagement Indicators served as dependent variables: Discussions with Diverse Others, Supportive Environment, and Quality of Interactions. Discussions with Diverse Others represents how often students had substantial interactions with individuals from different 
backgrounds or with different views. Questions in this indicator ask how often students had discussions with people who differ in terms of race or ethnicity, economic background, religious beliefs, and political views. The Supportive Environment indicator summarizes how much the institution emphasizes services and activities that support student success in a variety of areas, including academics, learning support, diversity, social opportunities, overall well-being, nonacademic responsibilities, and attending campus activities and events that address important issues. The Quality of Interactions indicator is built on the notion that supportive and positive relationships with key agents of socialization on campus can promote student learning and success. Questions in this indicator ask students to rate (on a 7-point scale from poor to excellent) their interactions with other students, academic advisors, faculty, student services staff, and other administrative staff and offices. Information on the reliability and validity of these variables can be found in NSSE's (n.d.) psychometric portfolio. However, the Cronbach's alphas for our analytic sample were $.84, .79$, and .87 for Discussions with Diverse Others, Quality of Interactions, and Supportive Environment, respectively. While Engagement Indicators are scored by NSSE on a scale from 0 to 60 , we standardized these variables with a mean of 0 and standard deviation of 1 to efficiently compute effect size estimates.

Our key independent variable was taken from a question about how the students found or were matched with their roommates. This item was worded as follows:

\section{Which of the following best describes how you became roommates or housemates?}

$\square$ Matched after creating an electronic housing profile

Assigned by the housing office or property owner

Planned with friend(s)

Found through social media

Found through an advertisement, Craigslist, etc. 
After viewing the frequencies, we collapsed the response options into two categories: (a) students who were matched by their school (matched after creating a profile or assigned by the housing office), and (b) students who were not matched by their school (planned with friends, or found through social media or advertisement).

Our second key variable was the students' race/ethnicity, as provided by the institutions in their NSSE population files. Response frequencies made possible the following groups for analysis: Asian, Black, International, Latinx, Multiracial, White, and Other (including American Indian or Alaska Native, Native Hawaiian or Other Pacific Islander, Other, and Unknown). To control for confounding variables, we also utilized data on students' sex, major, estimated GPA, transfer status, educational aspiration, first-generation status, age, intercollegiate athletics participation, and enrollment status (full- or part-time). These variables were collected on the core NSSE instrument or reported by their institution.

\section{Analyses}

We began our analyses by examining the frequency with which students chose or were assigned their roommates and the cross-tabulations between roommate selection and race. We then computed $t$-tests to compare the group means for our three dependent variables by roommate selection method.

Next, we fitted a multivariate model using ordinary least squares (OLS) regression that examined the main effect of being assigned a roommate by the institution, controlling for the student characteristics previously described. We chose to use OLS despite the nesting of students within institutions because intraclass correlation coefficients were low $(<.03)$; however, we utilized robust standard errors to account for the independence assumption violation. 
Finally, we estimated an additional model for each dependent variable that included interaction terms between roommate match and race/ethnicity. The interaction terms allowed us to examine if the relationship between roommate selection and the dependent variable varied by race/ethnicity. For each model, we conducted a joint Wald test to see if the coefficients associated with the interaction term were jointly significantly different from 0 . If the Wald test was significant at $p<.05$, we computed the predicted value for each group (matched status crossed with the student characteristic) when other characteristics were held at their mean to observe how the relationships differed.

Because large sample sizes as used in this study often lead to statistically significant findings that are trivial in magnitude, we examined standardized mean difference effect sizes in our interpretation of results. This includes both the mean differences between standardized scores in the bivariate comparisons ( $t$-tests) and the regression coefficients. NSSE researchers recommend interpreting standardized mean differences on the Engagement Indicators as follows: small effects are $\geq 0.1$, medium effects are $\geq 0.3$, and large effects are $\geq 0.5$. A statistically significant result with an effect size $<0.1$ may be inconsequential or trivial (Rocconi \& Gonyea, 2015).

\section{Limitations}

Our study is subject to some limitations that should be taken into account while reviewing its results. First, our results are correlational, not causal, as casual research would require entirely randomized roommate assignments. As forcing students into a living arrangement would violate research ethics, it is not a feasible option. Second, our sample contains a set of institutions that chose to administer NSSE and participate in the living arrangements study. While care was taken to ensure that they broadly represented the population 
of institutions with on-campus housing, they may not be representative of the intended population in unobservable ways. Third, our study focused on students' race/ethnicity and interactional diversity, but other forms of diversity like religion, politics, and economic (not captured on NSSE) are important to student learning outcomes. We encourage future studies to focus on these forms of diversity. Previous studies on roommates have examined the race/ethnicity of each individual in roommate pairings (e.g., Mark \& Harris, 2012). Therefore, our results are not directly comparable. We intentionally deviated from this historical line of inquiry to focus on how a policy like prohibiting students from choosing their roommate influences outcomes.

\section{Results}

About two-thirds of our sample (66\%) received their roommate through a matching procedure operated by their institution. However, this proportion varied by race/ethnicity (see Table 1). White students (60\%) were less likely to be matched by their institution than the average student. In contrast, about $75 \%$ of Asian, Black, and Latinx students were matched by their institution, and the proportion for international students was even higher at $82 \%$.

Using a two-group $t$-test, we found no significant difference in Discussions with Diverse Others (our proxy for interactional diversity) between students who were matched by their institution $(M=.02, S D=.98)$ and those who chose to live with a friend or someone they met on social media $(M=-0.01, S D=1.01 ; t(12,942)=1.85, p=.063)$. However, students who were matched by their institution $(M=-0.02, S D=1.00)$ perceived a less supportive environment than their peers who lived with a friend or someone they found through social media $(M=0.06$, $S D=1.00 ; t(12,991)=-4.62, p<.001)$. Similarly, students who were matched by their institution ( $M=-0.02, S D=1.00)$ rated the quality of their interactions less positively than students who 
lived with friends or someone they found through social media $(M=0.05, S D=0.99 ; t(12,744)=$ $-3.63, p<.001)$.

In our multivariate analyses, choosing your roommate was negatively related to having discussions with diverse others, $b=-0.05, t(12,544)=-2.46, p=.014$, after holding other factors constant. However, choosing your roommate was positively correlated, $b=0.07, t(12,604)=3.43$, $p=.000$, with perceiving a supportive environment. The relationship between choosing your roommate and quality of interactions, $b=-0.04, t(12,360)=1.95, p=.051$, bordered on significance after controlling for other variables. The results for the other control variables are available upon request from the authors.

Next, we replicated the regression models but included interaction terms between the method of roommate selection and race/ethnicity. After estimating the models, we conducted a Wald test to examine if the coefficients of the interaction terms were jointly equal to 0 . For Discussions with Diverse Others, we did not observe a significant difference in the relationship between the method of roommate selection and race/ethnicity overall, $F(6,12,538)=1.95$, $p=.070$. (The coefficient for the 'Other' race interaction term was significant at $p<.05$, but given the small $\mathrm{N}$ of this category the result is most likely spurious). In contrast, we observed significant variation in the estimated relationship between method of roommate selection and Supportive Environment by race/ethnicity, $F(6,12,595)=2.25, p=.035$. We observed similar differences in the coefficients by race/ethnicity for Quality of Interactions, $F(6,12,354)=3.89$, $p<.001$.

Table 2 contains the predicted values for Supportive Environment and Quality of Interactions by roommate match status and race. The first two estimates in each panel compare the predicted mean for the group (holding all other covariates at their mean) to the mean of the 
average student. The third estimate in each panel compares the predicted means within the racial group.

The predicted values for Supportive Environment are also plotted in Figure 1. Among Whites, students who were not matched with a roommate by their institution perceived a more supportive environment than the average student (0.05 SDs). The difference between White students who did and did not choose their roommate (0.04 SDs) was borderline significant at $p=$ .057. Asian/Pacific Islander students who were matched with a roommate by their institution perceived a less supportive environment than the average student (0.19 SDs). However, the difference between Asian/Pacific Islander students who did and did not live with their friends was not significant. Black students who were matched with a roommate by their institution $(0.07$ SDs) perceived a less supportive environment than the average student. Conversely, Black students who chose their roommates perceived a more supportive environment on average $(0.16$ SDs). The difference in means between Black students who were and were not matched by their institution was significant (0.23 SDs). Latinx students who were matched with a roommate by their institution perceived a more supportive environment than the average student (0.14 SDs). We did not observe significant differences for Latinx students who were not matched relative to the average student, nor for the within Latinx comparison. The mean for multiracial students who chose their roommates $(0.11$ SDs) was marginally significant relative to the average student $(p=.055)$. The difference (0.15 SDs) between multiracial students who did and did not choose their roommates was significant. We did not observe any significant differences for students in the catch-all other racial category or for international students relative to the average student or within the racial group. 
The predicted means for Quality of Interactions are visually displayed in Figure 2. White students who were (0.09 SDs) and were not (0.08 SDs) matched with a roommate by their institution rated their interactions with other students, faculty, and staff as higher quality than the average student. However, the within-race mean difference was not significant. Asian/Pacific Islander students who were matched with a roommate rated the quality of their interactions as poorer than the average student (0.14 SDs), but the result for students who chose their roommates was not significantly different from the average student. However, the difference in rating between Asian students who did and did not choose their roommate was significant $(0.24$ SDs). Black students who were matched with a roommate rated the quality of their interactions lower than the average student (0.35 SDs). The difference between Black students who chose their roommate was not significant relative to the average student. Nonetheless, the difference between Black students who did and did not choose their roommate was significant (0.25 SDs). We observed no significant differences for Latinx students. Multiracial students who were matched with a roommate rated their interactions poorer than the typical student (0.09 SDs). The difference between multiracial students who were and were not matched with a roommate was also significant (0.15 SDs). No significant differences were observed for students in the catch-all 'other' category. International students rated their quality of interactions poorer relative to the average student (0.09 SDs). However, the difference relative to students who chose their roommate was not significant.

\section{Discussion}

Our changing technological landscape has altered how students are paired with their roommate(s) (Conley, 2011; Li-A-Ping, n.d.; Ward, 2008; Washington Post Editors, 2010). In the past, students were limited to knowing just a fraction of their peers in their incoming class. 
Today, social media and other websites allow students to meet a substantial proportion of their peers before matriculating to college and more first-year students are choosing their roommates (Bauer-Wolf, 2018). This change has concerned some residence life professionals as students are likely to choose a roommate like themselves, which limit students' likelihood of interacting with peer students from different backgrounds and inhibiting their learning (Simon, 2018).

Consequently, some institutions, notably Duke, have decided to prevent their incoming students from selecting their first-year roommate. Yet, other research has demonstrated that incidents like microaggressions are common in spaces like residence halls and being subject to such incidents can negatively harm minority students' academic progress and learning through creating a negative campus climate (e.g., Solórzano, Allen, \& Carroll, 2002; Solórzano, Ceja, \& Yosso, 2000; Yosso, Smith, Ceja \& Solórzano, 2009). Due to these competing lines of inquiry, we investigated how the method of roommate choice influences interactional diversity and perceptions of the campus environment.

Our results start with an interesting and unexpected finding that White students were much more likely to choose their roommate - as much as 14 to 16 percentage points more than students of color. Several factors could explain this. First, White students were the largest racial/ethnic group in our sample, and with a few exceptions were attending predominantly White institutions. They may have had access to more friends and acquaintances from high school or their home community who also enrolled at the institution. It is also possible that White students, on average, have better access to social media and other social and cultural resources to find their roommates in advance.

Results for the Discussions with Diverse Others indicator, our proxy for interactional diversity, suggested that being matched with a roommate has a slight positive relationship with 
interactional diversity, but the magnitude of the difference was trivial or inconsequential. We expected students who were matched by the institution to be more engaged in discussions with others who differ from them, but the $t$-test result found no differences and the multivariate model found only a trivial effect (-0.05) in the expected direction (i.e., match students were slightly more likely to engage). This may be because our study only examined the roommate relationship without measuring associations with others within proximity in the residence hall. Indeed, the residence hall social environment includes many others living on the same floor area or building, and with whom they share common spaces such as dining halls, lounges, study areas, and recreation rooms. Diverse interactions can also happen in those places independent of the roommate relationship and are consistent with the social structure notion of proximity.

Similarly, our results for the Supportive Environment and Quality of Interactions indicators, our proxy for students' perceptions of the campus environment, did not vary meaningfully by the method of roommate assignment. Our multivariate results found a significant, but a trivial association for the method of roommate assignment for the Supportive Environment indicator and a non-significant relationship for Quality of Interactions.

However, we found that the relationship between the method of roommate selection and the Supportive Environment and Quality of Interactions indicators varied in non-trivial ways by race/ethnicity after controlling for other factors. The results for White, Latinx, and international students mirrored the overall results. However, we observed significant differences for Asian, Black, and multi-racial students. The most substantial differences from the overall results were for Black students. The effect size difference for Black students who chose their roommate and their peers who were matched by their institution was roughly 0.25 SDs. Thus, Black students who chose their roommate perceived a healthier campus environment than Black students who 
did not. We observed the same trend for multiracial students, but the magnitude of the difference was 0.15 SDs for both outcomes. Among Asian students, the difference in the Quality of Interactions variable for students who chose their roommate and their same race peers who did not was 0.24 SDs. However, the difference for the Supportive Environment indicator was nontrivial (0.15 SDs) for Asians, but was not statistically significant. These findings suggest that Asian, Black, and multiracial students tend to perceive a healthier campus environment when they choose their roommates, rather than relying on institutional matching. A likely reason for these findings is that rooms occupied by students of the same race act as counterspaces that embrace the norms of their cultural communities (Yosso et al., 2009).

When our results are viewed in combination, a few themes emerge. First, relying on the institution to provide a roommate has a trivial effect on promoting interactional diversity in contrast to other studies that have found policies like diversity coursework, intergroup relations experiences, or increasing structural diversity will have a more profound effect on interactional diversity (Bowman, 2010; Pike \& Kuh, 2006; Zúñiga, Nagda, Chesler, \& Cytron-Walker, 2007). Second, students of color who chose their roommate perceived a more supportive campus environment than their same race peers who were matched by their institution. Importantly, these differences were of such a magnitude that institutional matching may harm the educational opportunities of students of color. Thus, while policies like Duke's that prevent students from choosing their roommates are well-intentioned, they may suffer from unintended consequences that negatively impact the experiences of marginalized students. The notion that student affairs professionals must balance competing interests when changing policies is not novel. However, as Chang (2000) has noted, they are particularly fraught when they involve race. 
Our findings have a variety of implications for policy and practice. Our results suggest that students of color particularly benefit from choosing their roommate; yet, students of color are less likely to choose their roommates than White students. We are unable to determine the reason for the differential rates of choosing your roommate, but speculate that it could be due to some combination of a lack of feeder school networks from majority-minority high schools, differential rates of engagement in social media groups or websites for incoming students, and the acquisition of cultural capital associated with college knowledge. Consequently, institutions should take efforts to connect incoming students from affinity groups before their arrival on campus. Such efforts could include prospective student days or orientation targeted at students of color or have welcoming ceremonies for new students in their home communities. Alternately, institutions or students could create social media groups for incoming student affinity groups. A second implication is that our findings comport with Hallisey, Harren, \& Caple's (1980) belief that students are in a better place to choose their roommate than institutions. Consequently, institutions should generally not discourage students from choosing their roommate(s). Third, our findings underscore the importance of research and assessment when implementing policy change. Duke's policy changes, an impetus for this study, seem to be informed by the literature on interactional diversity, but not informed by the emerging literature on cultural engaging campuses and counterspaces. Thus, our findings reinforce the need to test out policy changes on small samples to measure both intended and unintended effects and/or consult various lines of inquiry about a policy. Fourth, our results highlight that policies can have disparate effects on student groups. In many studies, Latinx students are grouped with Black students under a label of underrepresented minority students. Yet, in the case of our results, Latinx students responded more similarly to White students than Black students. Similarly, Asian 
students, more commonly grouped with White students, had results more in concert with Black students.

\section{Conclusion}

Changes in technology have altered the proportion of first-year students who choose their own roommates (Bauer-Wolf, 2018). Student affairs professionals have become concerned that this change may have deleterious effects on students' interactional diversity, which impacts important student outcomes (Simon, 2018). In this study, we investigated this concern and also examined how the method of roommate selection influences perceptions of the campus environment. We found that the concerns over interactional diversity are largely unfounded. However, our results indicate the method of roommate selection is a contributing factor in how Asian, Black, and multiracial students perceive the campus environment. Therefore, our findings underscore the importance of evaluating how policy changes influence minority students' experiences. 


\section{References}

Antonio, A. L., Chang, M. J., Hakuta, K., Kenny, D. A., Levin, S., \& Milem, J. F. (2004). Effects of racial diversity on complex thinking in college students. Psychological Science, 15(8), 507-510.

Bauer-Wolf, J. (2018, March 2). Random roommates only. Inside Higher Ed. Retrieved from https://www.insidehighered.com/news/2018/03/02/duke-university-blocks-studentspicking-their-roommates-freshman-year

Blau, P. M. (1977). A macrosociological theory of social structure. American Journal of Sociology, 83, 26-34.

Bowman, N. A. (2010). Disequilibrium and resolution: The nonlinear effects of diversity courses on well-being and orientations toward diversity. The Review of Higher Education, 33(4), 543-568.

Chang, M. J. (1999). Does racial diversity matter?: The educational impact of a racially diverse undergraduate population. Journal of College Student Development, 40(4), 377-395.

Chang, M. J. (2000). Improving Campus Racial Dynamics: A Balancing Act Among Competing Interests. The Review of Higher Education 23(2), 153-175

Conley, D. (2011, August 28). When roommates were random. New York Times. Retrieved from https://www.nytimes.com/2011/08/29/opinion/when-roommates-were-random.html

Festinger, L., Schachter, S., \& Back, K. (1950). Social pressures in informal groups: A study of human factors in housing. Stanford University Press, Stanford.

Gurin, P., Dey, E., Hurtado, S., \& Gurin, G. (2002). Diversity and higher education: Theory and impact on educational outcomes. Harvard Educational Review, 72(3), 330-367. 
Gurin, P., Nagda, B. R. A., \& Lopez, G. E. (2004). The benefits of diversity in education for democratic citizenship. Journal of Social Issues, 60(1), 17-34.

Hallisey, J. N., Harren, V. A., \& Caple, R. (1980). Roommate changes in residence halls. Can they be predicted? The Journal of College and University Student Housing, 10(2), 7-9.

Holland, P. W., \& Lienhardt, S. (1972). Some evidence on the transitivity of positive interpersonal sentiment. American Journal of Sociology, 72, 1205-1209.

Hurtado, S., \& Carter, D. (1997). Effects of college transition and perceptions of the campus racial climate on Latina/o college students' sense of belonging. Sociology of Education, 70, 324 -345. http://dx.doi.org/10.2307/2673270

Li-A-Ping, J. (n.d.). 3 apps to find the perfect roommate. The Teen Entertainment \& Life Skills Multimedia Network. Retrieved from http://www.iaam.com/magazine/college/3-Apps-toFind-the-Perfect-Roommate

Mark, N. P. \& Harris, D. R. (2012). Roommate's race and the racial composition of white college students' ego networks. Social Science Research, 41(2), 331-342.

Molina, D. K., Heiselt, A., \& Justice, C. (2015). From matchmaker to mediator: Shifting trends in roommate relationships in an era of individualism, diversity, conflict, and social media. Journal of College and University Student Housing, 41(2), 104-116.

Museus, S. D. (2014). The Culturally Engaging Campus Environments (CECE) Model: A new theory of college success among racially diverse student populations. In M. B. Paulsen (Ed.), Higher education: Handbook of theory and research (pp. 189 -227). New York, NY: Springer. 10.1007/978-94-017-8005-6_5 
Museus, S. D., \& Maramba, D. C. (2011). The impact of culture on Filipino American students' sense of belonging. Review of Higher Education: Journal of the Association for the Study of Higher Education, 34, 231-258. http://dx.doi.org/10.1353/rhe .2010.0022

Museus, S. D., Yi, V., \& Saelua, N. (2017). How Culturally Engaging Campus Environments Influence Sense of Belonging in College: An Examination of Differences Between White Students and Students of Color. Journal of Diversity in Higher Education. Advance online publication. http://dx.doi.org/10.1037/dhe0000069

National Survey of Student Engagement. (n.d.). NSSE's Commitment to Data Quality. Retrieved from http://nsse.indiana.edu/html/psychometric_portfolio.cfm

Pike, G. R., \& Kuh, G. D. (2006). Relationships among structural diversity, informal peer interactions and perceptions of the campus environment. The Review of Higher Education, 29(4), 425-450.

Rocconi, L., \& Gonyea, R. M. (2015, May). Contextualizing student engagement effect sizes: An empirical analysis. Paper presented at the Association for Institutional Research Annual Forum, Denver, CO.

Simon, S. (2018). Why Duke University won't honor freshman roommate requests this fall. National Public Radio, Retrieved from https://www.npr.org/2018/04/21/602270265/whyduke-university-wont-honor-freshman-roommate-requests-this-fall

Solórzano, D., Allen, W. R., \& Carroll, G. (2002). Keeping race in place: A case study of racial microaggressions and campus racial climate at the University of California, Berkeley. UCLA Chicano/Latino Law Review, 23, 15-111. 
Solórzano, D., Ceja, M., \& Yosso, T. (2000). Critical race theory, racial microaggressions, and campus racial climate: The experiences of African American college students. The Journal of Negro Education, 69(1/2), 60-73.

Ward, B. J. (2008, December 18). There's something going down on Facebook. Pay attention [Blog post]. Retrieved from http://squaredpeg.com/index.php/2008/12/18/facebook-payattention/

Washington Post Editors. (2010). Facebookgate, the 2010 edition [Blog post]. Retrieved from http://voices.washingtonpost.com/campusoverload/2010/02/_admissions_officers_a_bit.html

Zúñiga, X., Nagda, B. A., Chesler, M., \& Cytron-Walker, A. (2007). Intergroup dialogue in higher education: Meaningful learning about social justice. ASHE-ERIC Higher Education Report, Vol. 32, No. 4. San Francisco: Jossey-Bass. 
Table 1.

Percentage distribution of roommate matching process by race/ethnicity

\begin{tabular}{lrr} 
& $\begin{array}{r}\text { Matched by } \\
\text { institution }\end{array}$ & $\begin{array}{r}\text { Friends or } \\
\text { Social } \\
\text { Media }\end{array}$ \\
\hline Asian & $76 \%$ & $24 \%$ \\
Black & $76 \%$ & $24 \%$ \\
Latinx & $74 \%$ & $26 \%$ \\
White & $60 \%$ & $40 \%$ \\
Multiracial & $70 \%$ & $30 \%$ \\
Other & $76 \%$ & $24 \%$ \\
International & $82 \%$ & $18 \%$ \\
Total & $66 \%$ & $34 \%$ \\
\hline
\end{tabular}


Table 2.

Predicted values by roommate match status and race

\begin{tabular}{|c|c|c|c|c|c|c|c|c|c|c|c|c|}
\hline & \multicolumn{6}{|c|}{ Supportive Environment } & \multicolumn{6}{|c|}{ Quality of Interactions } \\
\hline & \multicolumn{6}{|c|}{ Not } & \multicolumn{6}{|c|}{ Not } \\
\hline & Matched & & Matched & & $\Delta$ & & Matched & & Matched & & $\Delta$ & \\
\hline White & 0.01 & & 0.05 & $* *$ & -0.04 & $\dagger$ & 0.09 & $* * *$ & 0.08 & $* * *$ & 0.01 & \\
\hline Asian & -0.19 & $* * *$ & -0.04 & & -0.15 & & -0.14 & $* *$ & 0.10 & & -0.24 & $* *$ \\
\hline Black & -0.07 & $*$ & 0.16 & $* *$ & -0.23 & $* *$ & -0.35 & $* * *$ & -0.10 & & -0.25 & $* * *$ \\
\hline Latinx & 0.14 & $* *$ & 0.05 & & 0.09 & & 0.03 & & 0.00 & & 0.03 & \\
\hline Multiracial & -0.04 & & 0.11 & $\dagger$ & -0.15 & $*$ & -0.09 & $*$ & 0.06 & & -0.15 & $*$ \\
\hline Other & -0.03 & & -0.12 & & 0.10 & & -0.15 & & -0.25 & & 0.10 & \\
\hline International & -0.03 & & 0.02 & & -0.05 & & -0.09 & $*$ & -0.04 & & -0.06 & \\
\hline
\end{tabular}

Note: Matched = Roommate assigned by the institution; Not matched = Roommate chosen by the student; $\Delta=$ within racial/ethnic group difference; the results are when sex, major, estimated GPA, transfer status, educational aspiration, first-generation status, age, intercollegiate athletics participation, and enrollment status (full- or part-time) are held at their means.

$\dagger p<.10, * p<.05, * * p<.01, * * * p<.001$ 
Figure 1.

Predicted values of Supportive Environment by race and roommate match status

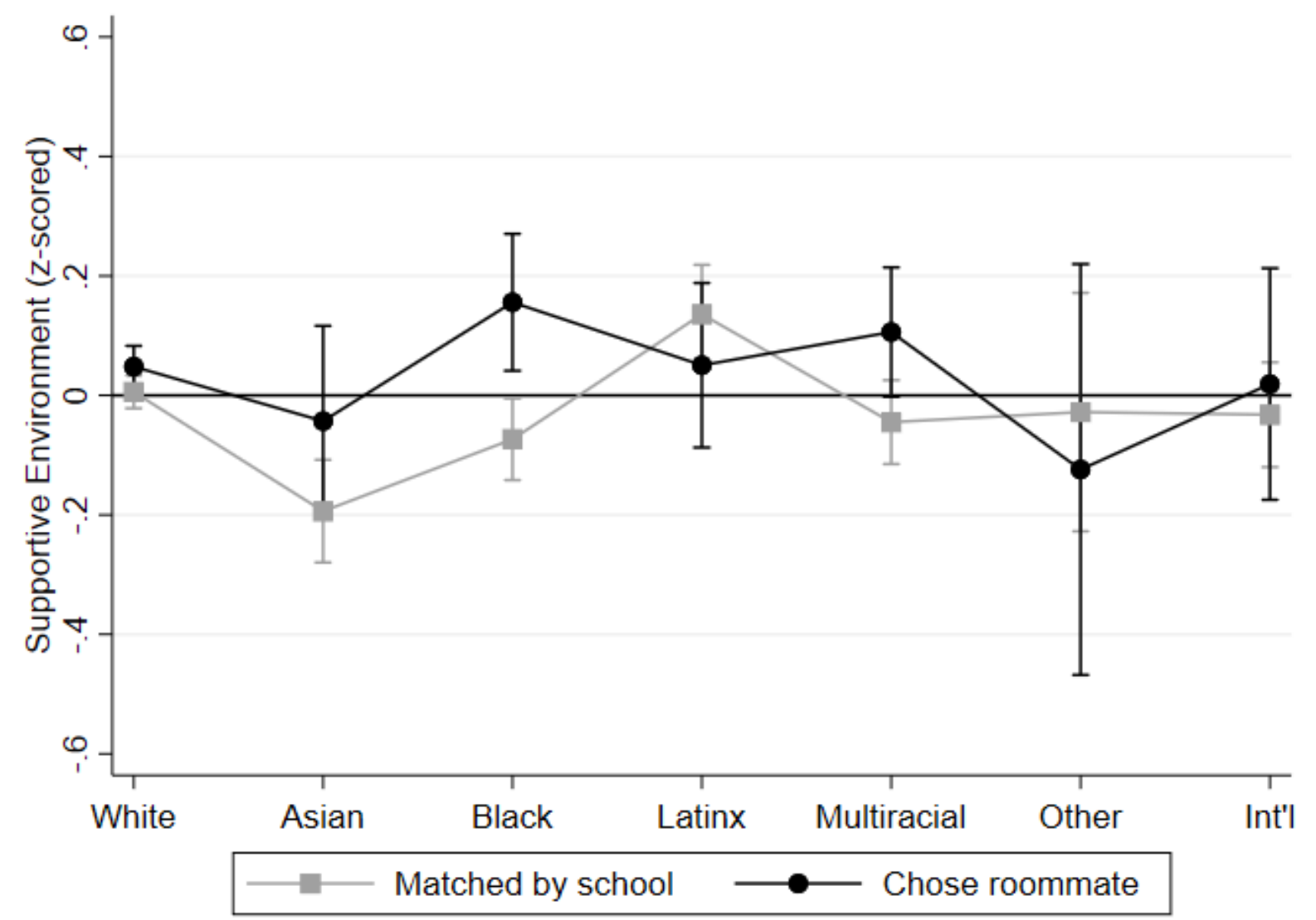

Note: All other covariates held at their mean 
Figure 2.

Predicted values of Quality of Interactions by race and roommate match status

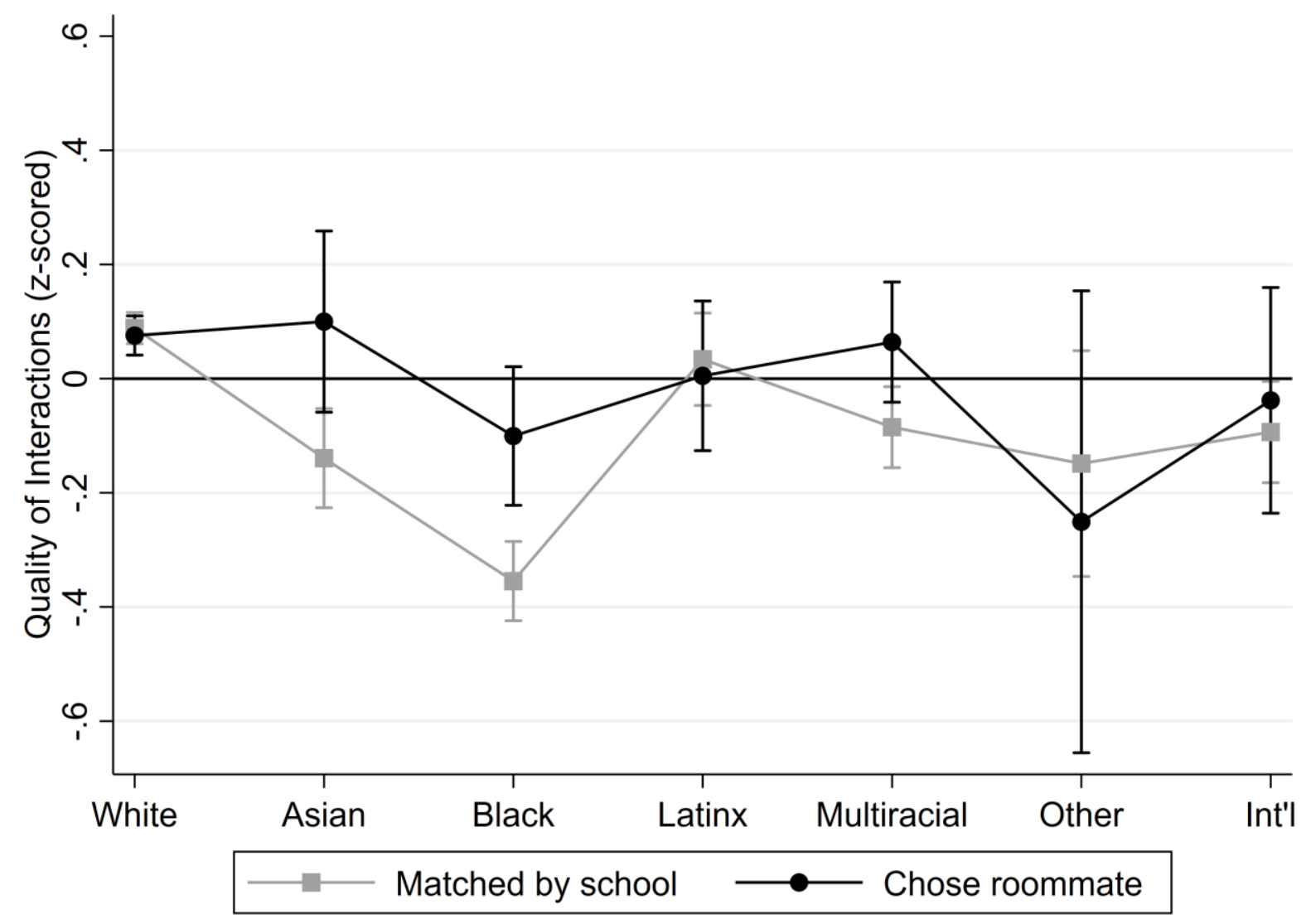

Note: All other covariates held at their mean 